\section{OC-040 A SYSTEMATIC APPROACH TO THERAPEUTIC TARGET SELECTION IN GASTRO-ESOPHAGEAL ADENOCARCINOMA}

\author{
doi:10.1136/gut.2011.239301.40
}

\begin{abstract}
A L Paterson, ${ }^{1}$ N B Shannon, ${ }^{1}$ P Lao-Sirieix, ${ }^{1, *}$ C J Peters, ${ }^{1}$ M O'Donovan, ${ }^{2}$ R C Fitzgerald ${ }^{1}$ Hutchison-MRC Research Centre, Cambridge, UK; ${ }^{2}$ Department of Pathology, Addenbrooke's Hospital, Cambridge, UK
\end{abstract}

Introduction The future success of individualised, molecular targeted therapy will depend on identification and thence inhibition of the oncogene(s) on which that tumour is dependent for survival. Gastro-esophageal tumours are common, lethal and molecularly heterogeneous. Currently chemotherapy relies on generic regimens and neo-adjuvant treatment only affords an extra 3.5 months survival compared to surgery alone.

Aims To identify the most active signalling pathways and compare different therapeutic strategies (upstream and downstream inhibitors) to induce cytotoxicity.

Methods Gene set enrichment analysis (GSEA) using KEGG annotated pathways was utilised to identify the most enriched pathways in a gene expression dataset from 75 chemo-naive gastro-oesophageal tumours. For independent validation Erk (total and phosphorylation levels) was used as a read-out for MAPK pathway activation and applied to a tissue microarray containing cores from 434 cases. The identity of the receptor tyrosine kinase(s) (RTK) responsible for MAPK activation was ascertained using a RTK array on 46 snap frozen samples for gastro-oesophageal tumours. The effects of Inhibition of active RTK(s) on proliferation, apoptosis and phosphorylation of downstream MAPK components were tested in 5 cell lines (OE33, OE19, MKN45, HSC39 and KATOIII). The most effective RTK inhibitor(s) were compared to downstream inhibition of Mek.

Results MAPK was the pathway statistically enriched in the greatest proportion of samples (32/75 samples, $42.7 \%)$ and 148/434 (34.3\%) tumour cores displayed immunostaining for phosphorylated Erk. Based on RTK arrays, the most frequently activated RTKs likely to account for this were members of the EGFR and FGFR family as well as c-met. No RTKs were active in $8 / 46$ cases $(17.4 \%)$, EGFR alone was active in $8 / 46$ cases (17.4\%), 2 receptors, usually members of the EGFR family, were active in $13 / 46$ cases $(28.3 \%)$ and multiple RTKs (median of 9) were active in 16/46 cases (37.0\%). Targeted inhibition of RTK(s) based on their activation profile led to inhibition of cellular proliferation, increased apoptosis and abrogation of downstream signalling components in vitro inall 5 lines. The most cytotoxic combination of inhibitors was cell line dependent and could not be predicted easily from the RTK activity profile. Inhibition of the downstream MAPK pathway component Mek induced equivalent or better cytotoxicity in all cell lines.

Conclusion We have shown that the MAPK pathway is commonly activated and can be effectively silenced either by identifying and inhibiting key RTKs or via use of a single drug for the downstream hub Mek. The efficacy of targeting a downstream fragile point warrants testing in clinical trials.

Competing interests None.

Keywords gastro-oesophageal tumour, MAPK pathway, targeted therapy. 